\title{
Individual behavior at habitat edges may help populations persist in moving habitats
}

\author{
Jane S. MacDonald • Frithjof Lutscher
}

Received: date / Accepted: date

\begin{abstract}
Moving-habitat models aim to characterize conditions for population persistence under climate-change scenarios. Existing models do not incorporate individual-level movement behavior near habitat edges. These smallscale details have recently been shown to be crucially important for large-scale predictions of population spread and persistence in patchy landscapes. In this work, we extend previous moving-habitat models by including individual movement behavior. Our analysis shows that populations might be able to persist under faster climate change than previous models predicted. We also find that movement behavior at the trailing edge of the climatic niche is much more important for population persistence than at the leading edge.
\end{abstract}

Keywords moving habitat model; edge behavior; linear analysis; eigenvalue approximation; population persistence

Mathematics Subject Classification (2000) 92D40 · 35K57

\section{$1 \quad 1$ Introduction}

2 As the earth's climate is warming, the geographic locations of climatic niches for various species shift towards higher latitude and/or altitude. The affected

This work was supported by an NSERC Discovery Grant (RGPIN-2016-0495) to FL.

Jane S. MacDonald

Department of Mathematics and Statistics, University of Ottawa, Ottawa, ON, Canada

Tel.: ++16135625800

Fax: ++1 613-562-5776

E-mail: jmacd142@uottawa.ca

Frithjof Lutscher

Department of Mathematics and Statistics, and Department of Biology, University of Ottawa, Ottawa, ON, Canada

Tel.: ++1 6135625800 ext. 3510

Fax: ++1 613-562-5776

E-mail: flutsche@uottawa.ca 
4 populations have to either adapt to new climatic conditions or move to new geographic locations with their climatic niches (Walther et al., 2002). For species with limited dispersal capacity, it might be challenging to follow their climatic niche, in particular in northern latitudes where climate change is already observed and still predicted to manifest more drastically than elsewhere (IPCC Working Group I, 2007). Several mathematical models have explored the relationship between a population's dispersal ability and growth capacity and the maximal movement speed of their climatic niche that still allows the population to persist (Potapov and Lewis, 2004; Berestycki et al., 2009; Zhou and Kot, 2011; Harsch et al., 2014). We contribute to this body of knowledge by including a more detailed description of individual movement behavior into the model.

The original model by Potapov and Lewis (2004) and virtually all related models since, conceptualize the suitable habitat of a species, as defined by appropriate climatic conditions, by a bounded interval on the real line, representing latitudinal coordinates from the equator to the pole. Inside the suitable habitat, the population has a positive intrinsic growth rate, outside the population declines. The bounded interval moves along the real line at constant speed to represent the movement of the climatic niche, hence the classification as 'moving-habitat model' (Harsch et al., 2017). A typical result is that if the speed of the climatic niche is small, then the population can persist, but if it is large, the population becomes extinct. In the simplest case, namely when there is no Allee effect, this result can be obtained from studying the stability properties of the trivial solution, i.e. the absence of a population. Among other aspects that previous studies consider are: the outcome of competition (Potapov and Lewis, 2004), the shape of the population distribution (Berestycki et al., 2009), the effect of different dispersal patterns and discrete generations (Zhou and Kot, 2011, 2013), impacts of stage structure in the population (Harsch et al., 2014), the effects of a gradient in growth rate (Li et al., 2014), and the possibility of gap formation (Berestycki et al., 2014). Models with Allee effect were studied, numerically and analytically by Berestycki and Rossi (2008, 2009); Roques et al. (2008); Bouhour and Nadin (2015).

Some, but not all, of the above mentioned models allow the movement behavior of individuals to depend on whether the individual is in suitable or unsuitable habitat. None of the models consider other behavior, such as habitat preference at an edge of a suitable patch. Yet, many empirical studies document different movement behavior in different habitat types as well as habitat preference and edge behavior of insects, birds, and mammals (Crone and Schultz, 2008). And recent theoretical work underlines the importance of including these details into reaction-diffusion models to obtain correct estimates for persistence conditions and spreading speeds (Maciel and Lutscher, 2013, 2015; Lutscher and Musgrave, 2017). More importantly, as Maciel and Lutscher (2013) point out, even if there is no preference at a habitat edge, as long as the movement behavior on the two sides of the edge is different, the standard mathematical assumptions of continuity of density cease to hold. All previous moving habitat models make this assumption. 
In this work, we generalize the (single-species) model by Potapov and Lewis (2004); Berestycki et al. (2009) to allow for differential movement behavior ahead and behind the suitable habitat, as well as habitat preference by individuals. These aspects lead us to consider discontinuous density-matching conditions across the edges of the suitable habitat (Section 2). We analyze the persistence conditions by studying a corresponding eigenvalue problem (Section 3). We illustrate how the critical speed and/or the length of the moving habitat depend on model parameters, in particular on diffusion rates outside the suitable habitat and habitat preferences (Section 4). In the final part of this work (Section 5), we consider an approximation of the dominant eigenvalue that determines population persistence in terms of residence times. Such an approximation was originally developed for symmetric problems (Cobbold and Lutscher, 2014), but the constant speed at which our habitat here moves introduces an asymmetry that makes the approximation fail. We find a more general method that improves the approximation in the symmetric case and allows an application to the asymmetric case.

\section{Model presentation}

Our model is a significant generalization of the model studied by Berestycki et al. (2009), which is a single-species version of the model by Potapov and Lewis (2004). Following these previous authors, we consider the population dynamics of a single species in an infinite, one-dimensional landscape. There is a suitable habitat patch of length $L>0$ that moves along the real line with constant speed $c \geq 0$, which corresponds to the velocity at which temperature isoclines move towards increasing latitude or altitude. We denote by $u(x, t)$ the density of the population at time $t>0$ and location $x \in \mathbb{R}$, and by $L_{1}(t)=c t$ and $L_{2}(t)=L+$ ct the boundary of the suitable patch. Inside the suitable patch, the population grows logistically with intrinsic growth rate $r$ and a constant coefficient for intraspecies competition, $a$. The diffusion constant is denoted by $D$. It is assumed that movement and growth happen on the same timescale. Thus, the equation in the suitable habitat is

$$
u_{t}=D u_{x x}+u(r-a u), \quad L_{1}(t)<x<L_{2}(t),
$$

where subscripts in $t, x$ denote partial derivatives with respect to time and space, respectively.

In the unsuitable habitats ahead and behind the suitable patch, the population dynamics are simply linear mortality and movement. We denote by $m_{1}$, $D_{1}$ and $m_{2}, D_{2}$ the mortality rate and diffusion coefficient to the left of $L_{1}(t)$ and to the right of $L_{2}(t)$, respectively. So, in the unsuitable habitats we have the equations

$$
\begin{aligned}
& u_{t}=D_{1} u_{x x}-m_{1} u, \quad x<L_{1}(t) \text {, } \\
& u_{t}=D_{2} u_{x x}-m_{2} u, \quad x>L_{2}(t) \text {. }
\end{aligned}
$$


All population dynamics parameters are assumed positive.

Finally, we need to impose matching conditions for the density and flux at each interface. We follow Ovaskainen and Cornell (2003); Maciel and Lutscher (2013), who derived such conditions from a random-walk model. We denote by $\alpha$ the probability with which an individual at the interface $L_{1}(t)$ decides to move into the suitable habitat, and by $\beta$ the corresponding probability at $L_{2}(t)$. Then the matching conditions for the density across each interface are

$$
\begin{aligned}
& u\left(L_{1}^{+}(t), t\right)=k^{\alpha} u\left(L_{1}^{-}(t), t\right), \\
& u\left(L_{2}^{-}(t), t\right)=k^{\beta} u\left(L_{2}^{+}(t), t\right),
\end{aligned}
$$

with

$$
k^{\alpha}=\frac{\alpha}{1-\alpha} \sqrt{\frac{D_{1}}{D}}, \quad k^{\beta}=\frac{\beta}{1-\beta} \sqrt{\frac{D_{2}}{D}} .
$$

Superscripts + and - denote the limit as $x$ approaches the interface from the right and left, respectively. Please note that Maciel and Lutscher (2013) derive an alternative form of $k^{\alpha}$ in which the fraction of the diffusion coefficients appears instead of their square root. We only consider the version with the square root here as the one without gave qualitatively similar results in Maciel and Lutscher (2013).

To match the flux across an interface, we note that it consists of two components: individuals cross an interface either due to diffusive self-movement or due to the deterministic movement of the interface. To see this, we consider a simpler situation with only one interface denoted by $L(t)=c t$ on the real line and with no population dynamics. Then the equations to the left of the interface are $u_{t}=D_{1} u_{x x}$, and to the right of the interface we have $u_{t}=D u_{x x}$. Since there are no population dynamics, the total mass must be conserved, i.e.

$$
\frac{\mathrm{d}}{\mathrm{d} t} \int_{\mathbb{R}} u(x, t) \mathrm{d} x=0 .
$$

Under the assumption that $u, u_{x} \rightarrow 0$ as $|x| \rightarrow \infty$ we calculate

$$
\begin{aligned}
\frac{\mathrm{d}}{\mathrm{d} t} \int_{\mathbb{R}} u(x, t) \mathrm{d} x & =\frac{\mathrm{d}}{\mathrm{d} t}\left(\int_{-\infty}^{c t} u(x, t) \mathrm{d} x-\int_{\infty}^{c t} u(x, t) \mathrm{d} x\right) \\
& =c u\left(c t^{-}, t\right)+\int_{-\infty}^{c t} u_{t}(x, t) \mathrm{d} x-c u\left(c t^{+}, t\right)-\int_{\infty}^{c t} u_{t}(x, t) \mathrm{d} x \\
& =c u\left(c t^{-}, t\right)+\int_{-\infty}^{c t} D_{1} u_{x x}(x, t) \mathrm{d} x-c u\left(c t^{+}, t\right)-\int_{\infty}^{c t} D u_{x x}(x, t) \mathrm{d} x \\
& =c u\left(c t^{-}, t\right)+D_{1} u_{x}\left(c t^{-}, t\right)-c u\left(c t^{+}, t\right)-D u_{x}\left(c t^{+}, t\right) .
\end{aligned}
$$

Hence, the correct flux-matching condition is 


$$
D_{1} u_{x}\left(c t^{-}, t\right)+c u\left(c t^{-}, t\right)=D u_{x}\left(c t^{+}, t\right)+c u\left(c t^{+}, t\right) .
$$

Previous authors had only considered the diffusive component of the flux, but since they assumed that the density is continuous, their results are not affected by this oversight.

All things considered, our model consists of the equations

$$
\begin{cases}u_{t}=D u_{x x}+u(r-a u), & L_{1}(t)<x<L_{2}(t), \\ u_{t}=D_{1} u_{x x}-m_{1} u, & x<L_{1}(t), \\ u_{t}=D_{2} u_{x x}-m_{2} u, & x>L_{2}(t), \\ u\left(L_{1}^{+}(t), t\right)=k^{\alpha} u\left(L_{1}^{-}(t), t\right), & L_{1}(t)=c t, \\ \left(D u_{x}+c u\right)\left(L_{1}^{+}(t), t\right)=\left(D_{1} u_{x}+c u\right)\left(L_{1}^{-}(t), t\right), & \\ u\left(L_{2}^{-}(t), t\right)=k^{\beta} u\left(L_{2}^{+}(t), t\right), & L_{2}(t)=L_{0}+c t, \\ \left(D u_{x}+c u\right)\left(L_{2}^{-}(t), t\right)=\left(D_{2} u_{x}+c u\right)\left(L_{2}^{+}(t), t\right) . & \end{cases}
$$

One difference between our and the previous models by Potapov and Lewis (2004); Berestycki et al. (2009) is that we allow the behavior ahead of the suitable patch to differ from that behind the patch. The more important difference is that we include edge behavior from Maciel and Lutscher (2013), which directly enters the matching conditions of the density. Since the movement of the habitat induces an advective component of the flux, the matching conditions of the flux contain the density (and not only its gradient). Hence, edge behavior also enters the flux matching conditions indirectly.

To make this model somewhat more tractable, we introduce the change of variable $x \mapsto x-c t$ that fixes the domain to $[0, L]$, but generates an advective term in the density. Then we non-dimensionalize the model and, using the same variable names for the non-dimensional quantities as before, arrive at the equations

$$
\begin{cases}u_{t}=u_{x x}+c u_{x}+u(1-u), & 0<x<L, \\ u_{t}=D_{1} u_{x x}+c u_{x}-m_{1} u, & x<0, \\ u_{t}=D_{2} u_{x x}+c u_{x}-m_{2} u, & x>L, \\ u\left(0^{+}, t\right)=k^{\alpha} u\left(0^{-}, t\right), & \left(u_{x}+c u\right)\left(0^{+}, t\right)=\left(D_{1} u_{x}+c u\right)\left(0^{-}, t\right), \\ u\left(L^{-}, t\right)=k^{\beta} u\left(L^{+}, t\right), & \left(u_{x}+c u\right)\left(L^{-}, t\right)=\left(D_{2} u_{x}+c u\right)\left(L^{+}, t\right) .\end{cases}
$$

In this notation, we now have

$$
k^{\alpha}=\frac{\alpha}{1-\alpha} \sqrt{D_{1}} \text { and } \quad k^{\beta}=\frac{\beta}{1-\beta} \sqrt{D_{2}} .
$$

Our model presents an idealized case in which (i) the suitable and unsuitable habitat are separated by a sharp edge, and (ii) organisms can detect the edge. In reality, we may see more gradual transition zones rather than sharp 
edges, depending on the scale of investigation. Also, organisms may not easily or directly detect edges, in particular those that are determined by climate. For example, the tree line, the altitudinal or latitudinal climatic delineation of the habitat where trees are able to grow, looks like a sharp edge when traced on a map, and when seen from a distance on a mountain range. Up close however, tree density responds to micro-climate and often shows a more gradual transition zone rather than a sharp edge. Many bird species rely on trees for nest sites and adjust their movement behavior at edges (Creegan and Osborne, 2005) so that they are rarely found outside wooded areas. When viewed from afar, the density of these birds may then exhibit a sharp drop at the tree line. Up close, again, bird and nest density may decline more gradually with tree density, and different bird species respond differently to forest edges (Kroodsma, 1984). Under a changing climate, the tree line will move. The birds will respond to multiple cues, climatic and otherwise, in multiple ways, but as long they rely on trees for nesting sites, they cannot move faster than the tree line, and hence might show a delayed response. While we believe that our model with a sharp edge and clear detectability can give important insights into population dynamics, future models should explore the effects of wider transition zones and/or indirect mechanism of detection.

\section{Stability analysis}

We focus our work on finding conditions under which a species can persist in the climate change scenario, and specifically on how movement behavior affects these persistence conditions. The population can persist if the zero steady-state is unstable. For that reason, we study the stability behavior of the trivial steady state of system (10).

\subsection{Linearizing at zero}

Linearizing the equations at $u^{*}=0$ and separating variables $u(x, t)=T(t) X(x)$ gives the equations $T(t)=e^{\lambda t} T(0)$ and

$$
\begin{array}{rlr}
X^{\prime \prime}+c X^{\prime}+X & =\lambda X, & 0<x<L, \\
D_{1} X^{\prime \prime}+c X^{\prime}-m_{1} X & =\lambda X, & x<0, \\
D_{2} X^{\prime \prime}+c X^{\prime}-m_{2} X & =\lambda X, & x>L,
\end{array}
$$

with interface conditions

$$
\begin{aligned}
X\left(0^{+}\right)=k^{\alpha} X\left(0^{-}\right), & \left(X^{\prime}+c X\right)\left(0^{+}\right)=\left(D_{1} X^{\prime}+c X\right)\left(0^{-}\right) \\
X\left(L^{-}\right)=k^{\beta} X\left(L^{+}\right), & \left(X^{\prime}+c X\right)\left(L^{-}\right)=\left(D_{2} X^{\prime}+c X\right)\left(L^{+}\right) .
\end{aligned}
$$


We now use a procedure originally employed by Ludwig et al. (1979) and since frequently used (Potapov and Lewis, 2004; Maciel and Lutscher, 2013) to reduce the problem on the infinite line to one on a bounded interval. For $x \notin(0, L)$ we have the characteristic polynomials

$$
D_{i} n_{i}^{2}+c n_{i}-\left(m_{i}+\lambda\right)=0
$$

with roots

$$
n_{i}^{ \pm}=\frac{-c \pm \sqrt{c^{2}+4\left(m_{i}+\lambda\right) D_{i}}}{2 D_{i}} .
$$

We impose the condition that $X \rightarrow 0$ as $|x| \rightarrow \infty$ and assume $|\lambda| \ll 1$ is near the stability boundary $\lambda=0$. Then we have $n_{i}^{+}>0>n_{i}^{-}$. Consequently, solutions outside the suitable habitat are of the form $X(x) \sim e^{n_{1}^{+} x}$ for $x<$ 0 , and $X(x) \sim e^{n_{2}^{-} x}$ for $x>L$. In particular, they satisfy the differential equations $X^{\prime}=n_{1}^{+} X$ for $x<0$, and $X^{\prime}=n_{2}^{-} X$ for $x>L$. These relations allow us to reduce the interface conditions to the boundary conditions

$$
\begin{aligned}
& X^{\prime}+c X=\gamma^{\alpha} X, \quad \text { at } x=0, \\
& X^{\prime}+c X=\gamma^{\beta} X, \quad \text { at } x=L,
\end{aligned}
$$

where

$$
\gamma^{\alpha}=\frac{D_{1} n_{1}^{+}+c}{k^{\alpha}}, \quad \gamma^{\beta}=\frac{D_{2} n_{2}^{-}+c}{k^{\beta}} .
$$

One could, of course, combine the terms $c X$ and $\gamma^{\alpha, \beta} X$ in the boundary conditions above. However, the expression $X^{\prime}+c X$ represents the flux across the boundary and $\gamma^{\alpha, \beta}$ are the proportionality factors. The analysis and interpretation of the results turn out easier if this physical fact is taken into account.

Unfortunately, equation (12) together with (19)-(20) constitute a nonstandard eigenvalue problem as the eigenvalue appears inside the boundary conditions through the dependency of $n_{i}^{ \pm}$on $\lambda$. We circumvent this problem by generalizing a theorem from Potapov and Lewis (2004).

\subsection{Steady states and their stability}

To obtain a steady state of system $(10)$, we set $u_{t}=0$. Just as in the previous section, the resulting problem on the infinite line may be converted to a problem on a bounded domain with generalized boundary conditions in the form 


$$
\begin{cases}u_{x x}+c u_{x}+u(1-u)=0, & 0<x<L, \\ u_{x}+c u=\gamma_{0}^{\alpha} u, & x=0, \\ u_{x}+c u=\gamma_{0}^{\beta} u, & x=L,\end{cases}
$$

where

$$
\gamma_{0}^{\alpha}=\frac{c+\sqrt{c^{2}+4 m_{1} D_{1}}}{2 k^{\alpha}} \text { and } \gamma_{0}^{\beta}=\frac{c-\sqrt{c^{2}+4 m_{2} D_{2}}}{2 k^{\beta}}
$$

are obtained from $\gamma^{\alpha, \beta}$ by setting $\lambda=0$.

Following Potapov and Lewis (2004), we associate to this steady-state problem the new time-dependent system

$$
\begin{cases}u_{t}=u_{x x}+c u_{x}+u(1-u), & 0<x<L, \\ u_{x}+c u=\gamma_{0}^{\alpha} u, & x=0, \\ u_{x}+c u=\gamma_{0}^{\beta} u, & x=L .\end{cases}
$$

Non-stationary solutions to system (10) are not equivalent to those of system (24), but their stationary solutions coincide and are given by system (22). Due to this relation, we can study the effects of small perturbations away from stationary solutions of both systems. The following theorem is a generalization of Theorem 3.1 by Potapov and Lewis (2004).

Theorem 1 (Stability) Let $u^{*}(x)$ be a solution of system (22), then $u^{*}(x)$ is a steady state solution for both (10) and (24). If $u^{*}(x)$ is linearly stable for (10) then it is also linearly stable for (24) and vice versa.

The proof of this theorem carries over from the proof by Potapov and Lewis (2004) with some modifications. We present it in the appendix for completeness. Instead, we point to the physical underpinning of the proof, which will also be important later in interpreting the results.

The composite parameters $\gamma^{\alpha, \beta}$ that relate the flux at the boundary to the density have several important properties. Since $\gamma^{\alpha}>0>\gamma^{\beta}$, the boundary is 'leaky' i.e. the net flux is pointing outward, and the net flux increases with $\left|\gamma^{\alpha, \beta}\right|$. This can be seen by considering the equation without population dynamics and integrating to obtain an equation for the total mass as

$$
\frac{\mathrm{d}}{\mathrm{d} t} \int_{0}^{L} u(x, t) \mathrm{d} x=-\left(\left|\gamma_{0}^{\beta}\right| u(L)+\gamma_{0}^{\alpha} u(0)\right)<0 .
$$

It turns out that $\gamma^{\alpha}$ is monotone increasing in $m_{1}, \lambda, c$ and decreasing in $D_{1}$ and $\alpha$. Similarly, $\left|\gamma^{\beta}\right|$ is monotone increasing in $m_{2}, \lambda$ and decreasing in $\beta$, but increasing in $D_{2}$ and decreasing in $c$. 


\subsection{The Critical Patch-Size}

To calculate the critical patch-size for model (10), we calculate the stability conditions for system (24), as they are equivalent by the theorem above. We linearize system (24) at $u=0$ and make the change of variable $v=u e^{c x}$. After separating variables, we obtain a regular Sturm-Liouville problem for the eigenfunction $X$ with eigenvalue $\lambda$, namely

$$
\begin{array}{rlrl}
X^{\prime \prime}-c X^{\prime}+X & =\lambda X, & 0<x<L, \\
X^{\prime} & =\gamma_{0}^{\alpha} X, & x & =0, \\
X^{\prime} & =\gamma_{0}^{\beta} X, & x & =L .
\end{array}
$$

When $\lambda=0$ the characteristic polynomial for equation (26) has the roots

$$
n_{0}^{ \pm}=\frac{c}{2} \pm \frac{\sqrt{c^{2}-4}}{2}
$$

We consider two cases, depending on the sign of the radicand in (29).

\section{Case 1: A negative radicand}

When $c<2$, the radicand in (29) is negative. This is the case considered in Berestycki et al. (2009); our treatment is similar. When $\lambda=0$, the secondorder problem in (26) is equivalent to

$$
X^{\prime}=Y, \quad Y^{\prime}=X^{\prime \prime}=-X+c Y .
$$

A solution that satisfies the boundary conditions corresponds to a trajectory in the $(X, Y)$-phase plane that starts on the positively-sloped line $Y=\gamma_{0}^{\alpha} X$ and reaches the negatively-sloped line $Y=\gamma_{0}^{\beta} X$ in an $x$-interval of exactly length $L$. The origin of the system is an unstable focus with trajectories spiraling in the clockwise direction (see Figure 1). In particular, all trajectories that start at $Y=\gamma_{0}^{\alpha} X$ will eventually reach the line $Y=\gamma_{0}^{\beta} X$. Hence, a critical patch-size exists.

For an explicit expression of the critical patch-size, we write solutions of (26)-(28) in the form

$$
X(x)=e^{\frac{c}{2} x}\left[A_{1} \cos \left(z_{0} x\right)+A_{2} \sin \left(z_{0} x\right)\right], \quad \text { with } \quad z_{0}=\frac{\sqrt{4-c^{2}}}{2} .
$$

From the boundary conditions, we obtain the defining equations for $A_{i}$ as

$$
\left\{\begin{array}{l}
A_{1}\left(\frac{c}{2}-\gamma_{0}^{\alpha}\right)+A_{2} z_{0}=0 \\
A_{1}\left[\left(\frac{c}{2}-\gamma_{0}^{\beta}\right) \cos \left(z_{0} L\right)-z_{0} \sin \left(z_{0} L\right)\right] \\
\quad+A_{2}\left[\left(\frac{c}{2}-\gamma_{0}^{\beta}\right) \sin \left(z_{0} L\right)+z_{0} \cos \left(z_{0} L\right)\right]=0 .
\end{array}\right.
$$




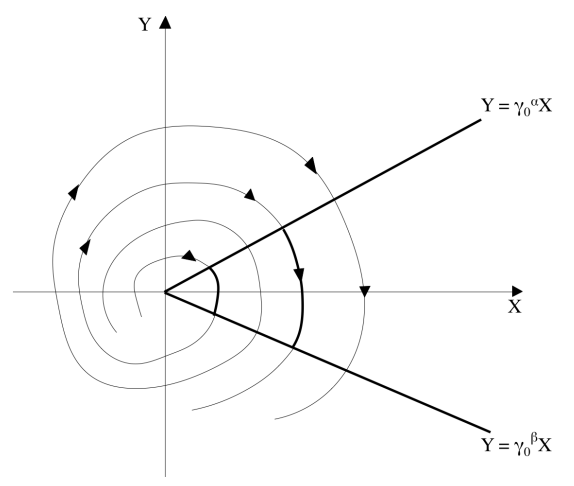

Fig. 1 Phase portrait of (30) for $c<2$. The origin is an unstable spiral. Every trajectory that starts at $Y=\gamma_{0}^{\alpha} X$ reaches $Y=\gamma_{0}^{\alpha} X$.

For a non-trivial solution, the determinant of the coefficient matrix of system (32) must be zero, which happens exactly if

$$
\sin \left(z_{0} L\right)\left[z_{0}^{2}+\left(\frac{c}{2}-\gamma_{0}^{\alpha}\right)\left(\frac{c}{2}-\gamma_{0}^{\beta}\right)\right]=\cos \left(z_{0} L\right) z_{0}\left(\gamma_{0}^{\alpha}-\gamma_{0}^{\beta}\right)
$$

Equivalently, we can write

$$
\tan \left(z_{0} L\right)=\frac{z_{0}\left(\gamma_{0}^{\alpha}-\gamma_{0}^{\beta}\right)}{z_{0}^{2}+\left(\frac{c}{2}-\gamma_{0}^{\alpha}\right)\left(\frac{c}{2}-\gamma_{0}^{\beta}\right)},
$$

whenever the denominator is not zero. We can solve for the critical patch-size $L^{*}$ in terms of model parameters as

$$
L_{c<2}^{*}=\frac{1}{z_{0}} \arctan \left(\frac{z_{0}\left(\gamma_{0}^{\alpha}-\gamma_{0}^{\beta}\right)}{z_{0}^{2}+\left(\frac{c}{2}-\gamma_{0}^{\alpha}\right)\left(\frac{c}{2}-\gamma_{0}^{\beta}\right)}\right) .
$$

Whenever $L \geq L_{c<2}^{*}$, the dominant eigenvalue $\lambda$ is positive and the zero steady-state is unstable; when $L<L_{c<2}^{*}$, then $\lambda$ is negative and the state is stable.

For numerical calculations, it is advantageous to evaluate condition (33) as to avoid erroneous results when the denominator in (35) becomes zero.

\section{Case 2: A positive radicand}

When $c \geq 2$, the radicand is non-negative. The equations for the vector field in the phase plane are the same as in the previous case. With the assumption $c \geq 2$, the eigenvalues $\sigma^{ \pm}$are real and positive with $\sigma^{+} \geq \sigma^{-}$. Thus, the origin is an unstable node. All trajectories will eventually increase to infinity along one of the directions given by the eigenvectors. 

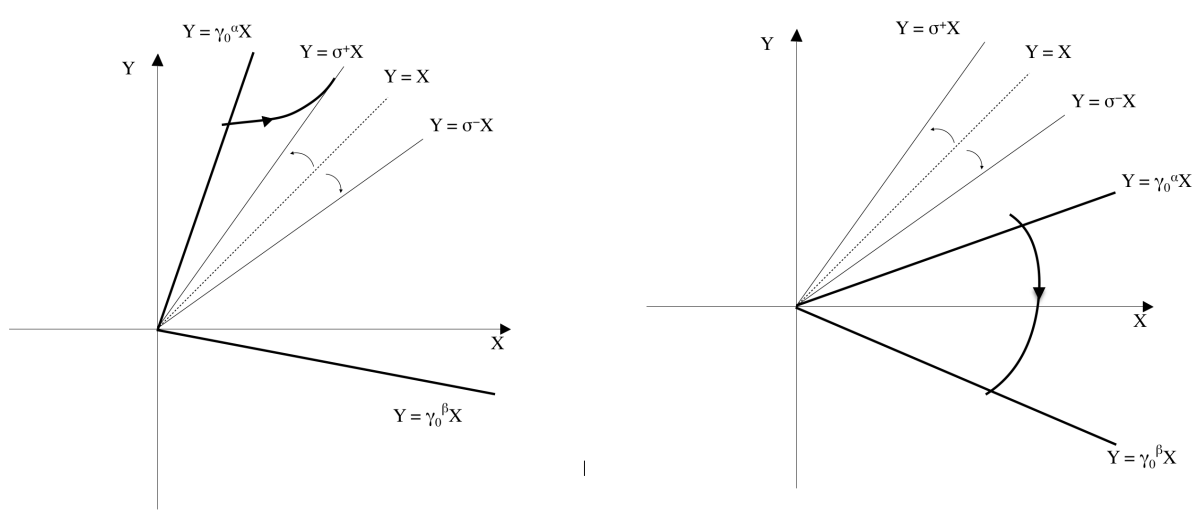

Fig. 2 Phase portrait for $c \geq 2$. When $\gamma_{0}^{\alpha}>\min \left\{\sigma^{ \pm}\right\}$, no connection can exist between the boundary conditions (left plot). When $\gamma_{0}^{\alpha}<\sigma^{ \pm}$, such a connection exists. The small arrows between the lines $Y=X$ and $Y=\sigma^{ \pm} X$ indicate that $\sigma^{+}$increases and $\sigma^{-}$decreases in $c$.

In the first quadrant below the line defined by $Y=\sigma^{-} X$, the vector field has directions $X^{\prime}>0$ and $Y^{\prime}<0$. In the fourth quadrant, the direction of the $X$-component changes to $X^{\prime}<0$, but the direction in the $Y$-component remains the same. Hence, trajectories that start in the first quadrant below $Y=\sigma^{-} X$, will eventually reach the axis $Y=0$ with $X>0$ and from there will eventually reach the line $X=0$ with $Y<0$.

The steepness of the boundary condition $Y=\gamma_{0}^{\alpha} X$ may be controlled by the parameter $k^{\alpha}$. Thus, for any fixed $c \geq 2$, we can choose $k^{\alpha}$ such that the boundary condition lies above or below the line $Y=\sigma^{-} X$. The former condition does not allow for a trajectory starting on the line $Y=\gamma_{0}^{\alpha} X$ to reach the line $Y=\gamma_{0}^{\beta} X$. As illustrated in the left plot figure 2, the path of a trajectory is obstructed by at least one of the eigenvectors. The latter condition does allow a trajectory to pass from one boundary condition to the other, see the right plot in figure 2 .

The necessary condition for the existence of a solution of the eigenvalue problem $(26)$ - $(28)$ is then

$$
\gamma_{0}^{\alpha}=\frac{c+\sqrt{c^{2}+4 m_{1} D_{1}}}{2 k^{\alpha}}<\sigma^{-}=\frac{c-\sqrt{c^{2}-4}}{2} .
$$

This condition can be formulated in terms of $k^{\alpha}$ as

$$
k^{\alpha}>\bar{k}^{\alpha}=\frac{c+\sqrt{c^{2}+4 m_{1} D_{1}}}{c-\sqrt{c^{2}-4}} .
$$

We summarize these considerations in terms of the critical patch-size as follows.

Theorem 2 (Existence of a Critical Patch Size for $c \geq 2$ ) For all $c \geq 2$, there exists a critical value $\bar{k}^{\alpha}$ as in (37) such that for all $\bar{k}^{\alpha}>\bar{k}^{\alpha}$ and $\bar{k}^{\beta}>0$ a finite critical patch-size $L^{*}=L^{*}\left(c, k^{\alpha}, k^{\beta}\right)$ exists. 
For an explicit representation formula of $L^{*}$ in the case $c \geq 2$, we write solutions to the linearized problem as

$$
X(x)=e^{\frac{c x}{2}}\left[A_{1} \cosh \left(s_{0} x\right)+A_{2} \sinh \left(s_{0} x\right)\right], \quad \text { with } \quad s_{0}=\frac{\sqrt{c^{2}-4}}{2} .
$$

From the boundary conditions we obtain the linear system

$$
\left\{\begin{array}{l}
A_{1}\left(\frac{c}{2}-\gamma_{0}^{\alpha}\right)+A_{2} s_{0}=0 \\
A_{1}\left[\left(\frac{c}{2}-\gamma_{0}^{\beta}\right) \cosh \left(s_{0} L\right)+s_{0} \sinh \left(s_{0} L\right)\right] \\
\quad+A_{2}\left[\left(\frac{c}{2}-\gamma_{0}^{\beta}\right) \sinh \left(s_{0} L\right)+s_{0} \cosh \left(s_{0} L\right)\right]=0 .
\end{array}\right.
$$

This system has a non-trivial solution under the condition

$$
\sinh \left(s_{0} L\right)\left[s_{0}^{2}+\left(\gamma_{0}^{\beta}-\frac{c}{2}\right)\left(\frac{c}{2}-\gamma_{0}^{\alpha}\right)\right]=\cosh \left(s_{0} L\right) s_{0}\left(\gamma_{0}^{\beta}-\gamma_{0}^{\alpha}\right),
$$

or equivalently, if the denominator does not vanish,

$$
\tanh \left(s_{0} L\right)=\frac{s_{0}\left(\gamma_{0}^{\beta}-\gamma_{0}^{\alpha}\right)}{s_{0}^{2}+\left(\gamma_{0}^{\beta}-\frac{c}{2}\right)\left(\frac{c}{2}-\gamma_{0}^{\alpha}\right)}
$$

Accordingly, we obtain the representation formula for the critical patch size to be

$$
L_{c \geq 2}^{*}=\frac{1}{s_{0}} \operatorname{arctanh}\left(\frac{s_{0}\left(\gamma_{0}^{\beta}-\gamma_{0}^{\alpha}\right)}{s_{0}^{2}+\left(\gamma_{0}^{\beta}-\frac{c}{2}\right)\left(\frac{c}{2}-\gamma_{0}^{\alpha}\right)}\right)
$$

\section{Illustrations}

\subsection{The Critical Patch-Size for $c<2$}

We begin by investigating the effects of the diffusion coefficients ahead $\left(D_{1}\right)$ and behind $\left(D_{2}\right)$ the suitable habitat. We assume that there is no habitat preference, i.e. $\alpha=\beta=0.5$, so that the discontinuity of the density across the interface is due only to a difference in diffusion rates.

The critical patch-size decreases with $D_{1}$ but increases with $D_{2}$, as the two plots in figure 3 indicate. As noted earlier, $\gamma_{0}^{\alpha}$ decreases with $D_{1}$ so that the flux out of the suitable patch decreases and hence the required length for persistence decreases. On the other hand, $\left|\gamma_{0}^{\beta}\right|$ increases with $D_{2}$, i.e. the flux out of the suitable habitat increases, and consequently a larger domain is required for persistence. 

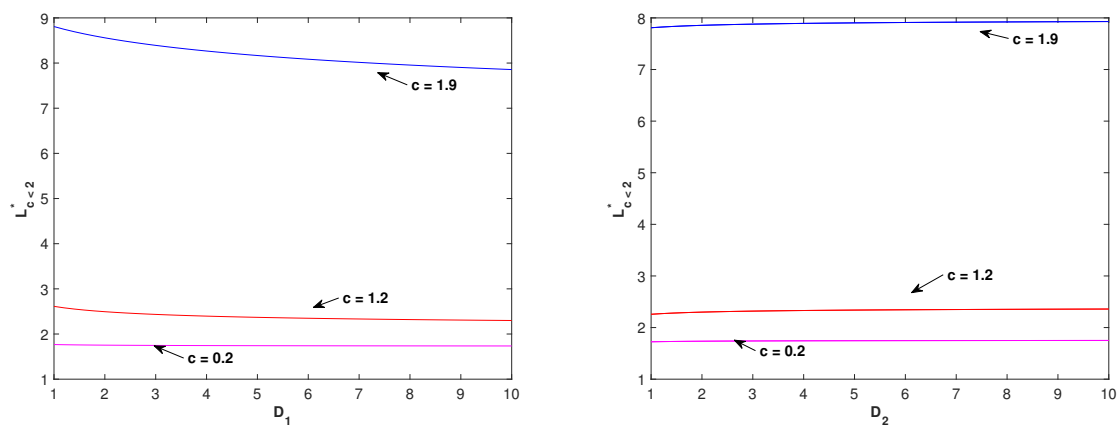

Fig. 3 The critical patch-size $L_{c<2}^{*}$ as a function of the parameter $D_{1}$ (left plot) and $D_{2}$ (right plot). Other parameters are $m_{i}=1.4, D_{i}=2$ and $\alpha=\beta=0.5$.

For a biological interpretation, we consider a randomly moving individual in the unsuitable habitat behind the trailing edge. If the interface moves fast (and away from the individual) and the individual moves slowly (randomly in both directions), then the individual will be further away from the suitable habitat over time and hence less likely to reach it again. Similarly, if an individual ahead of the suitable patch moves slowly (and randomly) and the interface moves quickly (and towards the individual), then the individual is likely to be swept up by the interface and back in the suitable patch. Thus, the critical patch-size is largest for low diffusion rates behind the trailing edge and high diffusion rates ahead of the patch. Vice versa, in a fast moving climate niche, fast diffusion behind the trailing edge and slow diffusion ahead increase the likelihood of persistence.

We also observe that the sensitivity of $L_{c<2}^{*}$ with respect to $D_{i}$, here defined as

$$
\left|\frac{\partial L_{c<2}^{*}}{\partial D_{i}}\right|
$$

decreases in $D_{i}$ and increases in $c$. These observations can be explained by the boundary conditions as before. In particular, $\gamma_{0}^{\alpha}$ is monotone decreasing in $D_{1}$ for each fixed $c>0$ and approaches $\sqrt{m_{1}}$ in the limit as $D_{1} \rightarrow \infty$. As a function of $c, \gamma_{0}^{\alpha}$ is increasing and the slope decreases with $D_{1}$.

Now we look at the effects of parameters $\alpha, \beta$, which denote the probability that an individual at the left-hand or right-hand interface will choose to move into the suitable habitat. We set $D_{1}=D_{2}=1$ so the discontinuity in density across an interface is due only to $\alpha, \beta \neq 0.5$

The critical patch-size $L_{c<2}^{*}$ is a decreasing function of $\alpha$, as is clear from figure 4 (left plot for $\beta=0.9$, right plot for $\beta=0.1$ ). Mathematically, as $\alpha$ increases to unity, $k^{\alpha}$ increases to infinity, and $\gamma^{\alpha}$ decreases to zero. Hence, the net outward flux at the trailing edge vanishes and the critical patch-size decreases. Biologically, as individuals increase their preference for the suitable patch, they are highly unlikely to leave this patch. And if individuals stay in the patch, the population is much more likely to persist. 

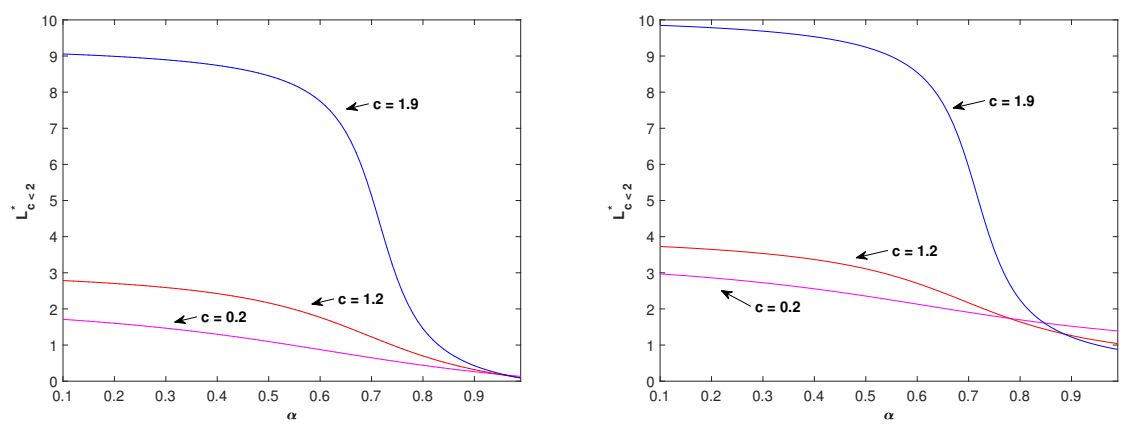

Fig. $4 L_{c<2}^{*}$ as a function of parameter $\alpha$. Other parameters are set to $m_{i}=1.4, D_{i}=1$ and $\beta=0.9$ (left plot) and $\beta=0.1$ (right plot).

We note that $L_{c<2}^{*}$ is more sensitive to changes in $\alpha$ when $c$ is larger. For fixed $c$, however, the sensitivity with respect to $\alpha$ is greatest for intermediate values of $\alpha$. We also note that $L_{c<2}^{*}$ is a decreasing function of $\beta$ (no plots provided).

The somewhat surprising observation is that $L_{c<2}^{*}$ is not monotone increasing in $c$ (for fixed $\alpha$ ). The curves corresponding to different values of $c$ intersect as $\alpha$ increases. For large values of $\beta$, these intersections happen for very large values of $\alpha$ and are only barely visible. They are clearly visible for smaller values of $\beta$.

To explain this observation, we note that the parameter $c$ affects $\gamma_{0}^{\alpha}$ as well as $\gamma_{0}^{\beta}$. Both values increase with $c$, but $\gamma_{0}^{\alpha}$ is positive and $\gamma_{0}^{\beta}$ is negative. Therefore, when $\left|\gamma_{0}^{\alpha}\right|$ is increasing, so is the net flux from the domain at $x=0$; when $\left|\gamma_{0}^{\beta}\right|$ is decreasing then so is the net flux from the domain at $x=L$. The total loss from the domain is the sum of the losses through each interface. When $\beta$ is large, the change in $\gamma_{0}^{\beta}$ with respect to $c$ is minimal and the increase in $\gamma_{0}^{\alpha}$ leads to the increased critical domain size. When $\beta$ is small, the change in $\gamma_{0}^{\beta}$ with respect to $c$ is significant so that the critical patch-size decreases when $\alpha$ is fixed close to unity.

For a biological interpretation, we consider an individual at the leading edge of the suitable habitat. If $\beta$ is large, then this individual is highly likely to stay in the suitable habitat, no matter how fast the habitat moves. The loss of individuals from the suitable habitat happens at the trailing end where a faster speed incurs a higher loss so that the critical patch size increases with c. On the other hand, if $\beta$ is small, then the individual at the leading edge is likely to leave the suitable patch. If the patch moves slowly, then the individual will move away and not return to the patch. If the patch moves fast, it is likely to catch up with the randomly moving individual and 'scoop it up' again. Even though the individual tries to leave $(\beta$ small) it cannot get away from the patch ( $c$ large) and therefore is not lost from the domain. Consequently, the critical size is small. 

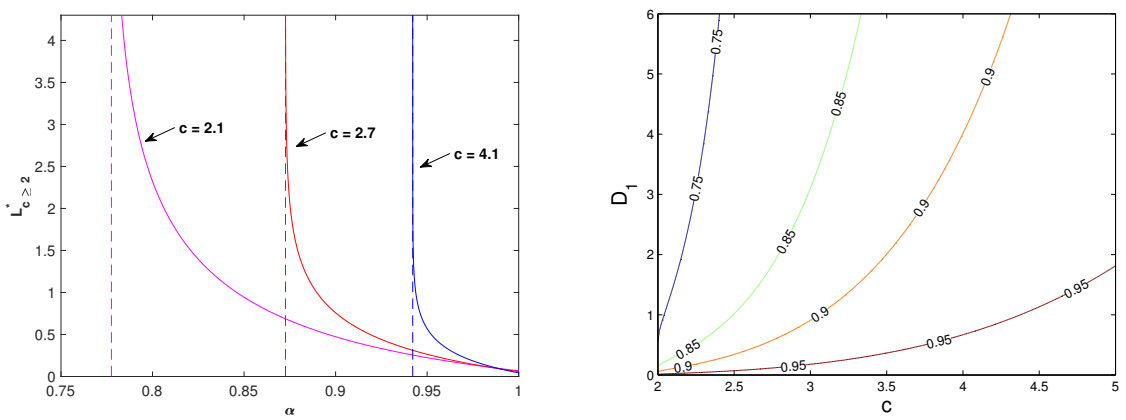

Fig. 5 The left plot shows $L_{c>2}^{*}$ as a function of the parameter $\alpha$. Model parameters are set to be $m_{1}=m_{2}=1.4, D_{1}=1.1, D_{2}=2$ and $\beta=0.9$. The solid lines are the plot of $L_{c>2}^{*}=L_{c>2}^{*}(\alpha)$. The dashed lines are the critical value $\alpha=\alpha^{*}$. The right plot shows the contours of $\alpha^{*}$ in the $c$ - $D_{1}$-plane.

\subsection{The Critical Patch-Size for $c \geq 2$}

When $c \geq 2$, the critical patch size is finite only if the condition in (37) holds, i.e. if

$$
k^{\alpha}>\frac{c+\sqrt{c^{2}+4 m_{1} D_{1}}}{c-\sqrt{c^{2}-4}} .
$$

This inequality can be re-written as a lower bound for $\alpha$ as

$$
\alpha>\alpha^{*}=\frac{I}{I+1}, \quad \text { with } \quad I=\frac{c+\sqrt{c^{2}+4 m_{1} D_{1}}}{\sqrt{D_{1}}\left(c-\sqrt{c^{2}-4}\right)} .
$$

As expected from the previous section, the critical patch-size $L_{c>2}^{*}$ is a decreasing function of $\alpha$, please see figure 5 . The explanation is the same as before: as $\alpha$ increases, fewer individuals leave the domain at the trailing edge, and therefore the population requires less space to persist. As $\alpha$ approaches $\alpha^{*}$ (indicated by the dashed line) from above, the critical patch-size approaches infinity. The surprising result that the critical patch-size is not an increasing function of the speed with which the patch moves arises here as well. The curves for different values of $c$ intersect.

The critical value $\alpha^{*}$ increases in $c$ and $m_{1}$ but decreases in $D_{1}$. The contour plot in Figure 5 reveals that $\alpha^{*}$ is, in general, more sensitive to $c$ than to $D_{1}$, except near the critical values $c=2$ and $D_{1}=0$. As $c$ approaches 2 , the critical value approaches

$$
\alpha_{\mid c=2}^{*}=\frac{1+\sqrt{1+m_{1} D_{1}}}{1+\sqrt{1+m_{1} D_{1}}+\sqrt{D_{1}}} .
$$

In Figure 5, this value is $\alpha_{\mid c=2}^{*} \approx 0.7121$.

As before, we note that $L_{c>2}^{*}$ is more sensitive to changes in $\alpha$ when $c$ is larger and less so when $\alpha$ is larger. Thus, as the speed of climate change 

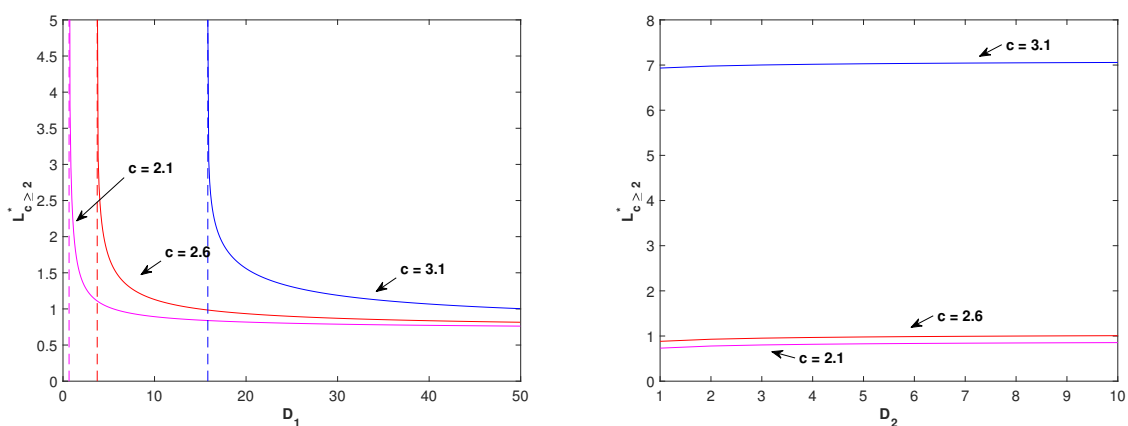

Fig. 6 The critical patch-size $L_{c \geq 2}^{*}$ as a function of the parameter $D_{1}$ (left plot) and $D_{2}$ (right plot). In the left plot, we have $\alpha=0.8$; on the right $\alpha=0.9021$. Other parameters are $m_{1}=1$ (left plot), $m_{1}=1.4$ (right plot), $m_{2}=1.4, D_{i}=1$ and $\beta=0.5$.

increases, it becomes increasingly important for individuals to detect the trailing edge of the suitable habitat and to adjust their movement behavior. If the patch is moving fast, individuals that leave the patch at the trailing edge have only a very small chance to ever catch up again. Hence, persistence is possible only if individuals do not leave the patch in the first place.

Just like in the case $c<2$ before (see Figure 3 ), the critical patch size for $c \geq 2$ is an increasing function of the diffusion rate ahead of the leading edge $\left(D_{2}\right)$ and a decreasing function of the diffusion rate behind the trailing edge $\left(D_{1}\right)$, as can be seen in Figure 6 . In fact, when $c \geq 2$, there is a lower threshold value $D_{1}^{*}$ below which the population cannot persist. This threshold is determined from condition (37), similarly to the threshold $\alpha^{*}$ in (45). Condition (37) can be written as

$$
\frac{\sqrt{D_{1}}}{c+\sqrt{c^{2}+4 m_{1} D_{1}}}>\frac{1-\alpha}{\alpha\left(c-\sqrt{c^{2}-4}\right)} .
$$

The left-hand side is an increasing function of $D_{1}$. The threshold $D_{1}^{*}$ is reached when the inequality is an equality. Since the expression on the left-hand side is bounded above by $1 / \sqrt{2 m_{1}}$, we can also formulate a threshold in terms of mortality behind the trailing edge as

$$
m_{1}<\frac{1}{4}\left(\frac{\alpha\left(c-\sqrt{c^{2}-4}\right)}{1-\alpha}\right)^{2} .
$$

\section{Approximations}

Since the stability conditions of the trivial steady-state are so important for the fate of the population, it is desirable to have various measures and approximations for the dominant eigenvalue that determines stability. Such approximations are particularly helpful if they can be evaluated from different and 
probably independent data sets or experiments. Cobbold and Lutscher (2014) developed a framework that allows one to relate the dominant eigenvalue to the mean occupancy time, i.e. the mean time that an individual spends in a given domain. Biologically, the relation between occupancy time and persistence is relatively simple: on average, an individual has to spend enough time in the domain to produce at least one offspring for the population to persist. Mathematically, the question is what the correct average is in a spatial model. The work by Cobbold and Lutscher (2014) treats this question for symmetric dispersal processes, but does not work well for the asymmetric dispersal that we have in model (10) due to the advective term. We briefly review the approach by Cobbold and Lutscher (2014) and then derive a novel formula that provides an improved approximation in the symmetric case and that works (to some extent) for asymmetric dispersal.

We work with the associated system in (24), since the theory by Cobbold and Lutscher (2014) is developed for bounded domains and since the stability behavior is the same as in (10). We linearize the equation and write the result as

$$
u_{t}=\mathcal{M}[u]+r u,
$$

with (scaled) growth rate $r=1$, where $\mathcal{M}$ consists of the differential operator

$$
\mathcal{M}[u](x)=u_{x x}+c u_{x}, \quad x \in \Omega=[0, L],
$$

and flux boundary conditions

$$
u_{x}(0)+c u(0)=\gamma_{0}^{\alpha} u(0), \quad u_{x}(L)+c u(L)=\gamma_{0}^{\beta} u(L) .
$$

The dominant eigenvalue of $\mathcal{M}$ is negative since the total density is decreasing, see (25). We denote it as $-\nu$ (with $\nu>0$ ) and the corresponding (positive) eigenfunction as $\phi$.

The trivial solution of (46) is unstable if $\nu<r=1$. Note that $\nu$ measures the loss rate of individuals due to movement out of the domain and $r=1$ is the growth rate. Hence, the persistence condition simply states that the reproduction rate has to be higher than the loss rate.

We want to relate $\nu$ to the mean occupancy time. We write $\mathcal{M}^{*}$ for the adjoint operator of $\mathcal{M}$ with respect to the standard inner product

$$
\langle f, g\rangle=\int_{\Omega} f(x) g(x) \mathrm{d} x .
$$

Its dominant eigenvalue is also $-\nu$; we denote the eigenfunction by $\psi$.

We denote the fundamental solution of (46), i.e. the solution with initial condition given by the Dirac distribution $u(0, x)=\delta(x-y)$, by $G(x, y, t)$. As in Cobbold and Lutscher (2014), we can express the probability that an individual initially located at $y \in \Omega$ is still in the domain at time $t$ as

$$
S(y, t)=\int_{\Omega} G(x, y, t) \mathrm{d} x .
$$




$$
\frac{1}{\nu} \frac{\phi(x)}{\bar{\phi}} \approx \int_{0}^{\infty} \int_{\Omega} G(x, y, t) \mathrm{d} y \mathrm{~d} t=\int_{\Omega} B(x, y) \mathrm{d} y=\int_{\Omega} B(y, x) \mathrm{d} y=T(x)
$$

The first passage probability $F(y, t)$, defined as the probability that an individual with initial location $y \in \Omega$ leaves $\Omega$ at time $t$, satisfies the equation

$$
\int_{0}^{t} F(y, t) \mathrm{d} t=1-S(y, t)
$$

With this, we can define the mean first passage time from initial location $y \in \Omega$ as

$$
T(y)=\int_{0}^{\infty} t F(y, t) \mathrm{d} t=\int_{0}^{\infty} \int_{\Omega} G(x, y, t) \mathrm{d} x \mathrm{~d} t,
$$

Strictly speaking, the mean first passage time is the time until the individual first leaves the domain. In our case, the boundary conditions take into account that the individual may leave and return several times during its lifetime. We therefore call this quantity the mean occupancy time. For a detailed discussion about this subtle difference, please see Cobbold and Lutscher (2014),

To calculate the mean occupancy time, it helps to introduce the occupancytime density

$$
B(x, y)=\int_{0}^{\infty} G(x, y, t) \mathrm{d} t
$$

which satisfies the equation $\mathcal{M} B=-\delta$, the Dirac distribution. We calculate

$$
\int_{\Omega} B(x, y) \mathrm{d} x=T(y)=-\int_{\Omega} T(x) \mathcal{M} B(\cdot, y) \mathrm{d} x=-\int_{\Omega} B(x, y) \mathcal{M}^{*} T \mathrm{~d} x .
$$

Hence, $T$ can be obtained from solving $\mathcal{M}^{*} T=-1$ in $\Omega$.

With this notation, we can explain how the dominant eigenvalue of $\mathcal{M}$ is related to the spatial average of $T$ and why this relation is only correct if movement is symmetric, i.e. if $G(x, y, t)=G(y, x, t)$. We denote the spatial average of the eigenfunction $\phi$ by $\bar{\phi}=\int_{\Omega} \phi(x) \mathrm{d} x /|\Omega|$. By definition, $\phi$ satisfies

$$
\phi(x) e^{-\nu t}=\int_{\Omega} G(x, y, t) \phi(y) \mathrm{d} y=\bar{\phi} \int_{\Omega} G(x, y, t) \mathrm{d} y+\int_{\Omega} G(x, y, t)(\bar{\phi}-\phi(y)) \mathrm{d} y .
$$

If we assume that the eigenfunction is reasonably close to its spatial average, we can neglect the last term. Since $\nu>0$, we can integrate the equality with respect to time and obtain The second last equality only holds if the movement process is symmetric. 1 Now we take averages on both sides and find 


$$
\frac{1}{\nu} \approx \bar{T}=\frac{1}{|\Omega|} \int_{\Omega} T(x) \mathrm{d} x .
$$

The following three ideas and observations allow us to improve the approximation above in such a way that it also extends to asymmetric dispersal. First, even though the assumption of a uniform distribution of the initial location of the individual in the averaging formula in (57) may be parsimonious, it does not seem to be the best. For example, with hostile boundary conditions, the assumption has a particularly large error at the boundary. Instead, at least at small population densities, the distribution is closer to the eigenfunction than to the constant. Secondly, for the dominant eigenfunction $\phi$ we have

$$
\nu\langle T, \phi\rangle=\langle T, \nu \phi\rangle=-\langle T, \mathcal{M} \phi\rangle=-\left\langle\mathcal{M}^{*} T, \phi\right\rangle=\langle 1, \phi\rangle .
$$

Tho Therefore, the weighted average of $T$ with weight function $\phi$ gives exactly the absolute value of the inverse of the eigenvalue:

$$
\bar{T}^{\phi}:=\frac{\int_{\Omega} T(x) \phi(x) \mathrm{d} x}{\int_{\Omega} \phi(x) \mathrm{d} x}=\frac{\langle T, \phi\rangle}{\langle 1, \phi\rangle}=\frac{1}{\nu}
$$

Note that in this notation, the expression in (57) is simply $\bar{T}=\bar{T}^{1}$.

Finally, the derivation in (56) suggests that $\phi$ is approximated (up to a constant) by

$$
R(x)=\int_{\Omega} B(x, y) \mathrm{d} y .
$$

Putting everything together, we suggest that $\nu$ is well approximated by the inverse of the weighted mean

$$
\nu \approx \frac{1}{\bar{T}^{R}}, \quad \text { where } \quad \bar{T}^{R}=\frac{\langle T, R\rangle}{\langle 1, R\rangle}=\frac{\int_{\Omega} T(x) R(x) \mathrm{d} x}{\int_{\Omega} R(x) \mathrm{d} x} .
$$

Function $R$ can be obtained by solving the equation $\mathcal{M} R=-1$ in $\Omega$.

Ballyk et al. (1998) derived the interpretation of $1 / \nu$ as the mean residence time in a different way directly from the decay rate of the corresponding eigenfunction. Our expression in (59) clarifies that this mean is a weighted mean of exit times with weight function equal to the dominant eigenfunction.

\subsection{The case of hostile exterior}

We return to our moving habitat model and apply the expression in (61) to approximate the persistence condition and demonstrate the validity of the formula as well as its limits. In general, the expressions are difficult to compute, but in a special case, all the formulas are relatively simple, namely when the unsuitable patches are completely hostile (i.e. $m_{i} \rightarrow \infty$ ) or, equivalently, if individuals at the boundary always leave the suitable patch (i.e. $\alpha=\beta=0$ ). Then we have 


$$
\mathcal{M}[u]=u_{x x}+c u_{x}, \quad u(0)=u(L)=0,
$$

and find the dominant eigenvalue and eigenfunction to be

$$
-\nu=-\frac{\pi^{2}}{L^{2}}-\frac{c^{2}}{4}, \quad \phi(x)=e^{-c x / 2} \sin (\pi x / L) .
$$

The adjoint operator $\mathcal{M}^{*}$ is the same as $\mathcal{M}$ with $c$ replaced by $-c$. Accordingly, the dominant eigenfunction $\psi$ is the same as $\phi$ with $c$ replaced by $-c$

The mean occupancy time in this case is the same as the mean first passage time. It satisfies the equation

$$
T^{\prime \prime}-c T^{\prime}=-1, \quad T(0)=T(L)=0,
$$

as derived above. Alternatively, the equation for $T$ can be derived from a random walk approach in a moving habitat in a similar fashion as McKenzie et al. (2009) derived it for a stationary habitat, see Appendix B.

The explicit expression for $T(x)$ is

$$
T(x)=\frac{1}{c^{2}}\left(1-e^{c x}\right)\left(T_{0}+c\right)+\frac{x}{c}, \quad T_{0}=\frac{L+\frac{1-e^{c L}}{c}}{1-e^{c L}} .
$$

The equation and explicit expression for $R(x)$ are the same as for $T$ with $c$ replaced by $-c$.

The two plots in Figure 7 demonstrate the validity of the approximation. The panel on the left compares the spatial shape of the eigenfunction $\psi$ with the weight function $R$, both scaled to have identical average equal to unity. The functions $\phi$ and $T$ are mirror symmetric images of $\psi$ and $R$ with respect to $x \mapsto L-x$. The panel on the right shows that the approximation of the eigenvalue $\nu$ using the weighted average as in (61) is much better than the uniform approximation from (57), at least when $c$ is small enough. When $c$ becomes much larger, the eigenfunctions become more and more skewed, and the approximation $R(x) \sim \phi(x)$ becomes increasingly worse so that none of the approximations work any more.

\subsection{The case of boundary behavior}

We come back to the case with general boundary conditions, where the eigenvalue problem is

$$
\mathcal{M}[u]=u_{x x}+c u_{x}=-\nu u, \quad \text { with } \quad\left\{\begin{array}{l}
u^{\prime}+c u=\gamma_{0}^{\alpha} u, x=0 \\
u^{\prime}+c u=\gamma_{0}^{\beta} u, x=L .
\end{array}\right.
$$

The eigenvalues are given implicitly by the equation

$$
\tan (z L)=\frac{z\left(\gamma_{0}^{\alpha}-\gamma_{0}^{\beta}\right)}{z^{2}+\left(\gamma_{0}^{\alpha}-c / 2\right)\left(\gamma_{0}^{\beta}-c / 2\right)}, \quad z=\frac{1}{2} \sqrt{c^{2}-4 \nu}
$$



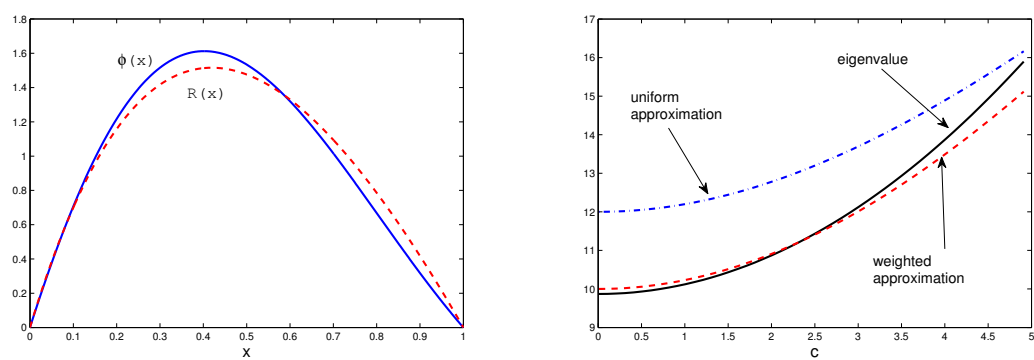

Fig. 7 Comparison of the approximations for the eigenfunction and the dominant eigenvalue for the case of hostile boundary conditions. Left plot: Function $R(x)$ approximates the eigenfunction $\phi(x)$ for $c=2$. Right plot: when $c$ is small enough, the true eigenvalue $\nu$ from (63) (solid) is approximated much better by the inverse of the weighted average in (61) (dashed) than by the uniform average in (57) (dash-dot). In both plots $L=1$.

and the corresponding eigenfunctions are

$$
\phi(x)=e^{-c x / 2}(\cos (z x)+B \sin (z x)), \quad B=\frac{\gamma_{0}^{\alpha}-c / 2}{z} .
$$

The function $R$ can be calculated from $\mathcal{M} R=-1$ as

$$
R(x)=-\frac{\zeta_{1}}{c} e^{-c x}-\frac{x}{c}+\zeta_{2},
$$

498 with

$$
\zeta_{1}=\frac{1+c\left(\gamma_{0}^{\alpha}-c\right) \zeta_{2}}{\gamma_{0}^{\alpha}} \quad \text { and } \quad \zeta_{2}=\frac{\gamma_{0}^{\alpha}\left(1+L\left(c-\gamma_{0}^{\beta}\right)\right)-\gamma_{0}^{\beta} e^{-c L}}{\gamma_{0}^{\alpha} c\left(c-\gamma_{0}^{\beta}\right)+c\left(\gamma_{0}^{\alpha}-c\right) \gamma_{0}^{\beta} e^{-c L}}
$$

The adjoint operator is not simply obtained by replacing $c$ with $-c$. Standard calculations give

$$
\mathcal{M}^{*}[u]=u_{x x}-c u_{x}, \quad \text { with }\left\{\begin{array}{l}
u^{\prime}=\gamma_{0}^{\alpha} u, x=0 \\
u^{\prime}=\gamma_{0}^{\beta} u, x=L .
\end{array}\right.
$$

The function $T$ that satisfies $\mathcal{M}^{*} T=-1$ is then

$$
T(x)=\frac{k_{1}}{c} e^{c x}+\frac{x}{c}+k_{2}
$$

with

$$
k_{1}=\frac{c \gamma_{0}^{\alpha} k_{2}-1}{c-\gamma_{0}^{\alpha}} \quad \text { and } \quad k_{2}=\frac{\left(\gamma_{0}^{\beta} L-1\right)\left(c-\gamma_{0}^{\alpha}\right)+e^{c L}\left(c-\gamma_{0}^{\beta}\right)}{\gamma_{0}^{\alpha} c e^{c L}\left(c-\gamma_{0}^{\beta}\right)+c \gamma_{0}^{\beta}\left(\gamma_{0}^{\alpha}-c\right)}
$$

The plots in Figures 8 and 9 illustrate the goodness of fit for the approximation via the weighted average and the gain compared to the uniform average for the eigenfunction and eigenvalue in two cases. Instead of the eigenvalue, 

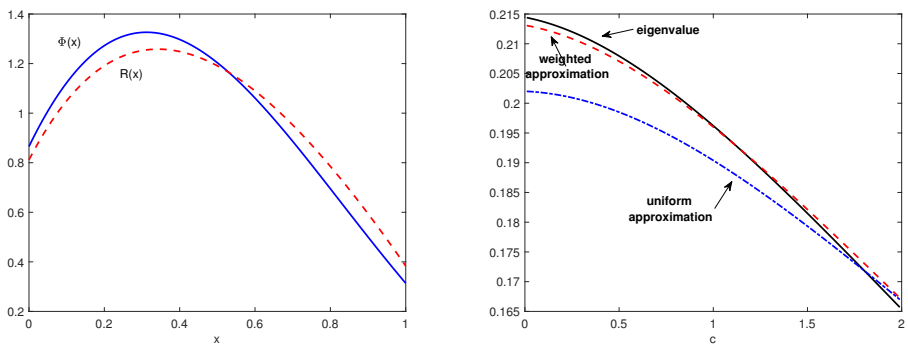

Fig. 8 Comparison of the approximations for the eigenfunction and the mean occupancy time for the case of general boundary conditions for small $\alpha$. Left plot: Function $R(x)$ approximates the eigenfunction $\phi(x)$ for $c=1.9$. Right plot: Mean occupancy time $1 / \nu$ (solid) and its uniform (dash-dot) and weighted (dashed) average approximations. In both plots, parameters are $m_{i}=D_{i}=1, \alpha=0.3, \beta=0.1$ and $L=1$.
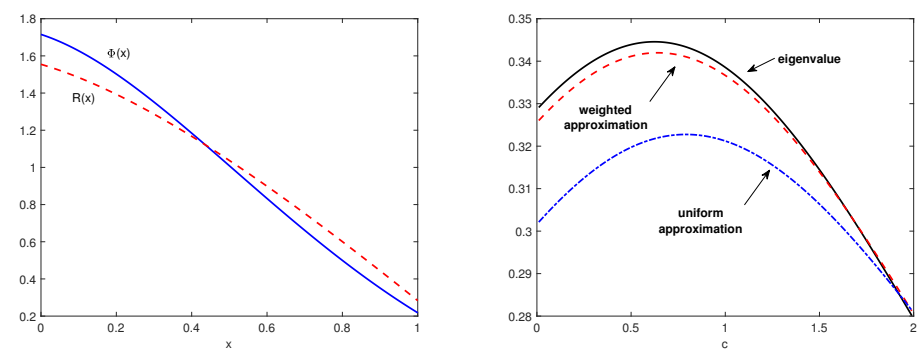

Fig. 9 Comparison of the approximations for the eigenfunction and the mean occupancy time for the case of general boundary conditions for large $\alpha$. Left plot: Function $R(x)$ approximates the eigenfunction $\phi(x)$ for $c=1.9$. Right plot: Mean occupancy time $1 / \nu$ (solid) and its uniform (dash-dot) and weighted (dashed) average approximations. In both plots, parameters are $m_{i}=D_{i}=1, \alpha=0.6, \beta=0.1$ and $L=1$.

we plot its inverse, the mean occupancy time. When $\alpha$ is relatively small, the eigenfunction $\phi$ as well as function $R$ are hump shaped as in Figure 8. On the other hand, if $\alpha$ is large, then both functions can be monotone decreasing as in Figure 9. Formally, the reason is that for these parameter values, we have $\gamma_{0}^{\alpha}<c$ and $R^{\prime}(0)=\left(\gamma_{0}^{\alpha}-c\right) R(0)<0$. Intuitively, the advective term will push individuals towards the trailing boundary, and if $\alpha$ is large, then individuals rarely leave the domain, so that the population is concentrating near the trailing edge. When $\alpha$ is large enough, we see that the mean occupancy time (and equivalently the dominant eigenvalue) are not monotone in $c$. We had seen earlier that the critical domain-size need not be monotone in $c$.

\section{Discussion}

The effects of climate change are visible in many ecosystems around the world. One such change is that optimal climatic conditions for many species shift to higher latitudes and/or altitudes. From a conservation perspective, one then 
needs to ask the question of whether a species can "keep pace with a shifting climate" (Berestycki et al., 2009). In their model, these authors let the growth conditions shift in space at a constant speed, but assume that individual dispersal is independent of growth and climatic conditions. These assumptions fit particularly for passively dispersed species, for example through windborne seeds. Active dispersers, on the other hand, can adjust their movement behavior to local conditions. A reasonable strategy would be to have small dispersal rates in good habitats (exploitation) and higher dispersal rates in less favourable environments (exploration). Potapov and Lewis (2004) in their moving-habitat model allowed for such a difference in dispersal rates between the suitable and unsuitable habitat. They assumed that dispersal ahead and behind the moving patch is identical. We argue that the conditions ahead and behind the moving suitable patch could be quite different (e.g. cooler ahead and warmer behind), so that dispersal behaviour could differ between these two regions. Furthermore, mortality rates could differ between these two regions as abiotic (e.g. climatic) and biotic (e.g. competition, predation) factors would differ. We allowed for these differences in our model.

More importantly, we included edge behavior in our model. Edge behavior is well documented for many taxa. Our approach is based on recent models for random walks near interfaces (Ovaskainen and Cornell, 2003; Maciel and Lutscher, 2013), but differs from these earlier papers in that our suitable patch is mobile. The population density in our model is not necessarily continuous across an interface between the suitable and unsuitable regions. Relatively abrupt changes of observed densities are observed in various taxa and are used as a basis for habitat suitability models and for projections of future species ranges (Leroux et al., 2013). In our model, this discontinuity appears from either of two factors: habitat preference and difference in diffusion rates. In that sense, some of the qualitative results in Potapov and Lewis (2004) should be revisited. We also clarified that the population flux across a moving boundary consists of two components, not only of the diffusive flux of individual movement.

Having all these individual-level details in our model allows us to tease apart the different influence of the different parameters and processes. In the classical minimal patch-size problem on an immobile patch (Skellam, 1951; Kierstead and Slobodkin, 1953), the population is more likely to persist when the growth rate is higher and the domain is longer, but less likely when diffusion is higher. For spatial spread, on the other hand, diffusion and growth rate are both positively related to invasion speed. On a moving-habitat model, we have a combination of critical patch-size and spread problems (Zhou and Kot, 2011). Clearly, an increase in growth rate and patch size or a decrease in mortality rates helps the population persist, and diffusion inside the suitable patch decreases the likelihood of persistence. Higher diffusion in front of the suitable patch marginally increases the critical patch-size whereas higher diffusion behind the suitable patch can considerably decrease these habitat requirements. Strong preference for the suitable habitat patch can obviously 
decrease the size requirement, but it turns out that preference at the trailing edge is much more important than at the leading edge.

Arguably the most surprising results of our investigation are with respect to the speed of the climatic niche. We found that a population may persist for speeds faster than the threshold speed that previous authors had found, provided the movement rates behind the trailing edge are high and/or the preference for the suitable patch is high. We also found that the critical patchsize is not necessarily monotone with respect to the speed of the climatic niche. While a faster moving patch 'scoops up' more individuals that dispersed ahead, it also loses more individuals behind. The net effect of these two processes can change sign.

Moving-habitat models are mathematically closely related to models for stream ecosystems (Pachepsky et al., 2005), where the habitat is fixed and the flow of water induces an advective term as long as the organism is not fully able to actively swim against the current. Our work could inspire similar research for population dynamics in streams, but the mechanisms underlying individual behavior would have to be carefully checked. For example, if an organism is a passive swimmer (so that the equations apply), the process of how it could change its behavior at boundaries of favourable habitat is not obvious (so that our interface conditions may not apply).

While edges of immobile habitats can be quite abrupt in many natural and human-managed landscapes, it is less likely that moving, climate-induced edges are equally sharp. Especially since climatic conditions around the long-term trend vary considerably between years, we expect more gradual transitions between suitable and unsuitable regions. We assumed that edges were localized and could be perceived by the organism. The more realistic assumption would be a more gradual transition. A first model in this spirit of environmental gradients was proposed and analyzed by Li et al. (2014). They considered a smooth monotone function representing habitat quality changing from negative to positive at the trailing edge of the species range (see also $\mathrm{Hu}$ and Zou (2017)). A habitat quality function that includes the leading and the trailing edge would have to be hump-shaped. Such a model was proposed in discrete time and without the effects of climate change by Latore et al. (1999) and then revisited and put into the moving-habitat context, in a stochastic setting by Zhou and Fagan (2017). However, these models assume that the movement of organisms is unbiased and unaffected by habitat or climatic conditions. Habitat-dependent movement could be included as a taxis term. This addition would make model analysis considerably more difficult. We believe that our model is a simplified first but useful and informative step in analyzing mechanisms that can help or hinder a population in keeping up with climate change.

In addition to or instead of behavioral responses, organisms may also evolve and adapt to changes in climatic conditions. The early landmark paper in this direction is by Pease et al. (1989) who model the spatial density and mean trait value along a spatial gradient. More recently, the question of how shifting habitats affect genetic diversity was studied by Garnier and Lewis (2016) 
with a reaction-diffusion model with shifting climate envelope. Their model is somewhat similar to ours but does not include boundary behavior or changes in diffusion rates. The authors conclude that fast moving habitats diminish diversity. It is conceivable that the same type of movement and boundary behavior that allows a population to persist at higher speeds in our model would preserve higher diversity in their setting.

There are numerous mathematical challenges arising from our work, most notably the generalization of the analytical results regarding eigenvalues and asymptotic behavior of the model on the real line by Berestycki et al. (2009) to our extended model. Similarly, including competition (Potapov and Lewis, 2004) and an Allee effect (Roques et al., 2008) would be challenging endeavours. Finally, a consumer-resource model could elucidate how a resource (e.g. vegetation) moves in response to climate change and what the emerging edges of the suitable patch of a consumer (e.g. herbivore) look like, before determining conditions under which the consumer persists in the system.

Acknowledgements The authors thank Gabriel Maciel for discussion and insights about the density and flux matching conditions at the boundary. FL would like to express his deep gratitude to $\mathrm{KPH}$ for his inspiration, guidance and role model in life.

\section{References}

Ballyk, M., Dung, L., Jones, D. A., and Smith, H. (1998). Effects of random motility on microbial growth and competition in a flow reactor. SIAM J. Appl. Math., 59(2), 573-596.

Berestycki, H. and Rossi, L. (2008). Reaction-diffusion equations for population dynamics with forced speed. I. The case of the whole space. Disc. Cont. Dyn. Sys. A, 21(1), 41-67.

Berestycki, H. and Rossi, L. (2009). Reaction-diffusion equations for population dynamics with forced speed. II. Cylindrical-type domains. Disc. Cont. Dyn. Sys. A, 25(1), 19-61.

Berestycki, H., Diekmann, O., Nagelkerke, C., and Zegeling, P. (2009). Can a species keep pace with a shifting climate? Bull. Math. Biol., 71(2), 399-429.

Berestycki, H., Desvillettes, L., and Diekmann, O. (2014). Can climate change lead to gap formation? Ecological Complexity, 20, 264-270.

Bouhour, J. and Nadin, G. (2015). A variational approach to reaction-diffusion equations with forced speed in dimension 1. Disc. Cont. Dyn. Sys. A, 35(5), 1843-1872.

Cantrell, R. S. and Cosner, C. (2003). Spatial Ecology via Reaction-Diffusion Equations. Mathematical and Computational Biology. Wiley.

Cobbold, C. and Lutscher, F. (2014). Mean occupancy time: linking mechanistic movement models, population dynamics and landscape ecology to population persistence. J. Math. Biol., 68, 549-579. 
Creegan, H. and Osborne, P. (2005). Gap-crossing decisions of woodland songbirds in Scotland: an experimental approach. Journal of Applied Ecology, 42, 678-687.

Crone, E. and Schultz, C. (2008). Old models explain new observations of butterfly movement at patch edges. Ecology, 89(7), 2061-2067.

Garnier, J. and Lewis, M. A. (2016). Expansion Under Climate Change: The Genetic Consequences. Bull. Math. Biol. 78, 2165-2185.

Harsch, M., Zhou, Y., HilleRisLambers, J., and Kot, M. (2014). Keeping pace with climate change: Stage-structured moving-habitat models. Am. Nat., 184(1), 25-37.

Harsch, M., Phillips, A., Zhou, Y., Leung, M.-R., Rinnan, D., and Kot, M. (2017). Moving forward: insights and applications of moving-habitat models for climate change ecology. J. Ecology, 105, 1169-1181.

$\mathrm{Hu}, \mathrm{H}$. and Zou, X. (2017). Existence of an extinction wave in the Fisher equation with a shifting habitat. Proc. Amer. Math. Soc., 145, 4763-4771.

IPCC Working Group I (2007). Projections of future changes in climate. IPCC Publications and Data. http://www.ipcc.ch/publications_and_data/ar4/wg1/en/spmsspmprojections-of.html.

Kierstead, H. and Slobodkin, L. B. (1953). The size of water masses containing plankton blooms. J. Marine Research, 12, 141-147.

Kroodsma, R. L. (1984). Effect of edge on breeding forest bird species. Wilson Bulletin, 96, 426-436.

Latore, J., Gould, P., and Mortimer, A. (1999). Effects of habitat heterogeneity and dispersal strategies on population persistence in annual plants. Ecolog. Modelling, 123, 127-139.

Leroux, S. J. and Larriveé, M. and Boucher-Lalonde, V. and Hurford, A. and Zuloga, J. and Kerr, J. T. and Lutscher, F. Mechanistic models for the spatial spread of species under climate change. Ecological Applications, 23, $815-828$

Li, B., Bewick, S., Shang, J., and Fagan, W. (2014). Persistence and spread of a species with a shifting habitat edge. SIAM J. Appl. Math., 74(5), 1397-1417.

Ludwig, D., Aronson, D. G., and Weinberger, H. F. (1979). Spatial patterning of the spruce budworm. J. Math. Biol., 8, 217-258.

Lutscher, F. and Musgrave, J. (2017). Behavioral responses to resource heterogeneity can accelerate biological invasions. Ecology, 98(5), 1229-1238.

Maciel, G. and Lutscher, F. (2013). How individual response to habitat edges affects population persistence and spatial spread. Am. Nat., 182(1), 42-52.

Maciel, G. and Lutscher, F. (2015). Allee effects and population spread in patchy landscapes. J. Biol. Dyn., 9, 109-123.

McKenzie, H., Lewis, M., and Merrill, E. (2009). First passage time analysis of animal movement and insights into the functional response. Bull. Math. Biol., 71(1), 107-129.

Ovaskainen, O. and Cornell, S. (2003). Biased movement at a boundary and conditional occupancy times for diffusion processes. J. Appl. Prob., 40(3), 
557-580.

Pachepsky, E., Lutscher, F., Nisbet, R., and Lewis, M. A. (2005). Persistence, spread and the drift paradox. Theor. Pop. Biol., 67, 61-73.

Pease, C. M. an Lande, R. and Bull, J. J. (1989) A Model of Population Growth, Dispersal and Evolution in a Changing Environment. Ecology, 70, 1657-1664.

Potapov, A. and Lewis, M. (2004). Climate and competition: The effect of moving range boundaries on habitat invasibility. Bull. Math. Biol., 66(5), 975-1008.

Roques, L., Roques, A., Berestycki, H., and Kretzschmar, A. (2008). A population facing climate change: joint influences of allee effects and environmental boundary geometry. Population Ecology, 50, 215-225.

Skellam, J. G. (1951). Random dispersal in theoretical populations. Biometrika, 38, 196-218.

Walther, G.-R., Post, E., Convey, P., Menzel, A., Parmesan, C., Beebee, T., Fromentin, J.-M., Hoegh-Guldberg, O., and Bairlein, F. (2002). Ecological responses to recent climate change. Nature, 416, 389-395.

Zhou, Y. and Fagan, W. (2017). A discrete-time model for population persistence in habitats with time-varying sizes. J. Math. Biol., 75(3), 649-704.

Zhou, Y. and Kot, M. (2011). Discrete-time growth-dispersal models with shifting species ranges. Theor. Ecol., 4, 13-25.

Zhou, Y. and Kot, M. (2013). Life on the move: Modeling the effects of climate-driven range shifts with integrodifference equations. In M. Lewis, P. Maini, and S. Petrovskii, editors, Dispersal, individual movement and spatial ecology, pages 263-292. Springer, Berlin.

\section{A Proof of Theorem 1.}

We deenote by $u^{*}(x)$ a non-negative, stationary solution for systems (10) and (24). Then the linearized equations of these two systems inside the interval $(0, L)$ are identical. We present the proof in two cases.

Case 1: $c=0$.

When $c=0$, the systems are governed by an elliptic, self-adjoint operator and consequently are known to have a principal eigenvalue that admits a positive eigenfunction (Cantrell and Cosner, 2003). The eigenvalue problem associated to the linearized system of (24) is

$$
\begin{cases}v_{x x}-g(x) v=\lambda v, & 0<x<L, \\ v_{x}-\gamma_{0}^{\alpha} v=0, & x=0, \\ v_{x}-\gamma_{0}^{\beta} v=0, & x=L,\end{cases}
$$

where $g(x)=2 u^{*}(x)-1, \gamma_{0}^{\alpha}=\frac{\sqrt{m_{1} D_{1}}}{k^{\alpha}}$ and $\gamma_{0}^{\beta}=-\frac{\sqrt{m_{2} D_{2}}}{k^{\beta}}$.

The eigenvalue problem corresponding to (10) is 


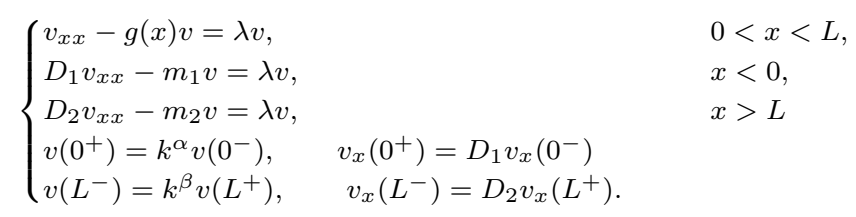

Consider the auxiliary quasi-eigenvalue problem corresponding to (73)

$$
\begin{cases}v_{x x}-g(x) v=\lambda v, & 0<x<L \\ D_{1} v_{x x}-m_{1} v=l v, & x<0 \\ D_{2} v_{x x}-m_{2} v=l v, & x>L\end{cases}
$$

with parameter $l>\max \left(-m_{1},-m_{2}\right)$ and interface conditions as in (73). The advantage of introducing the parameter $l$ is that upon using the same technique seen previously, (74) can be reduced to a system on a bounded domain, while excluding $\lambda$ from the boundary conditions. The resulting system is

$$
\begin{cases}v_{x x}-g(x) v=\lambda v, & 0<x<L, \\ v_{x}+B(x) u=0 & x=0, L,\end{cases}
$$

where

$$
B(x)=\left\{\begin{array}{lll}
-\frac{D_{1} \tilde{n}^{+}(l)}{k^{\alpha}}, & \text { at } & x=0, \\
-\frac{D_{2} \tilde{n}^{-}(l)}{k^{\beta}}, & \text { at } & x=L
\end{array}\right.
$$

and $\tilde{n}^{+}(l)=\sqrt{\frac{m_{1}+l}{D_{1}}}$ and $\tilde{n}^{-}(l)=-\sqrt{\frac{m_{2}+l}{D_{2}}}$. Corollary 2.2 in Cantrell and Cosner (2003) states that the principal eigenvalue of (75) is a continuous and decreasing function of $|B|$ and therefore also of $l$. We denote this eigenvalue as $\lambda(l)$.

1. Suppose that the principle eigenvalue $\lambda_{A}$ of (72) is positive. The function $\sigma(l)=\lambda(l)-l$ is continuously decreasing. We show that there exists some $l_{B}$ such that $0<l_{B}<\lambda_{A}$ and $\sigma\left(l_{B}\right)=0$. First, taking $l=0$ reduces (75) to (72). Thus $\sigma(0)=\lambda(0)=\lambda_{A}$. Second, for $l=\lambda_{A}$, we find $\sigma\left(\lambda_{A}\right)=\lambda\left(\lambda_{A}\right)-\lambda_{A}<\lambda(0)-\lambda_{A}=0$. Hence, $\sigma(0)>0>\sigma\left(\lambda_{A}\right)$. By the intermediate value theorem we have some $l_{B}$ with $\sigma\left(l_{B}\right)=0$, which implies $\lambda\left(l_{B}\right)=l_{B}$. Thus for $l=l_{B}$ system (74) is identical to (73) and hence there exists a positive eigenvalue $l_{B}$ of (73).

2. Now suppose that system (73) has a positive principal eigenvalue $\lambda_{B}>0$. Taking $l=\lambda_{B}$ implies that (75) has at least one positive eigenvalue, namely $\lambda_{B}$, in particular, its principal eigenvalue is then also positive. As $\lambda(l)$ is a decreasing function of $l$, we claim that $\lambda_{A}$ is also positive. Indeed, $\lambda_{A}=\lambda(0)>\lambda\left(l_{B}\right) \geq \lambda_{B}>0$.

Case 2: $c>0$.

When $c$ is non-zero, the operator governing these equations is no longer self-adjoint, but we can transform the system into a self-adjoint one by following chapter 2 of Cantrell and Cosner (2003).

The two eigenvalues to compare are

$$
\begin{cases}v_{x x}+c v_{x}-g(x) v=\lambda v, & 0<x<L, \\ v_{x}+c v=\gamma_{0}^{\alpha} v, & x=0, \\ v_{x}+c v=\gamma_{0}^{\beta} v, & x=L,\end{cases}
$$




$$
\begin{cases}v_{x x}+c v_{x}-g(x) v=\lambda v, & 0<x<L, \\ D_{1} v_{x x}+c v_{x}-m_{1} v=l v, & x<0, \\ D_{2} v_{x x}+c v_{x}-m_{2} v=l v, & x>L, \\ v\left(0^{+}\right)=k^{\alpha} v\left(0^{-}\right), \quad\left(v_{x}+c v\right)\left(0^{+}\right)=\left(D_{1} v_{x}+c v\right)\left(0^{-}\right), & \\ v\left(L^{-}\right)=k^{\beta} v\left(L^{+}\right), \quad\left(v_{x}+c v\right)\left(L^{-}\right)=\left(D_{2} v_{x}+c v\right)\left(L^{+}\right) . & \end{cases}
$$

The latter is equivalent to the eigenvalue problem on the bounded domain

$$
\begin{cases}v_{x x}+c v_{x}-g(x) v=\lambda v, & 0<x<L \\ v_{x}+c v=\gamma^{\alpha}(l) v, & x=0, \\ v_{x}+c v=\gamma^{\beta}(l) v, & x=L,\end{cases}
$$

753 with

$$
\gamma^{\alpha}(l)=\frac{D_{1} n_{1}(l)^{+}+c}{k^{\alpha}}, \quad \gamma^{\beta}=\frac{D_{2} n_{2}^{-}(l)+c}{k^{\beta}}, \quad \text { and } \quad n_{i}^{ \pm}(l)=\frac{-c \pm \sqrt{c^{2}+4\left(m_{i}+l\right) D_{i}}}{2 D_{i}} .
$$

755

The change of variable $w=v e^{c x}$ removes the advective term in the boundary conditions. Systems (77) and (79) become

$$
\begin{cases}w_{x x}-c w_{x}-g(x) w=\lambda w, & 0<x<L, \\ w_{x}=\gamma_{0}^{\alpha} w, & x=0, \\ w_{x}=\gamma_{0}^{\beta} w, & x=L,\end{cases}
$$

756 and

$$
\begin{cases}w_{x x}-c w_{x}-g(x) w=\lambda w, & 0<x<L \\ w_{x}=\gamma^{\alpha}(l) w, & x=0 \\ w_{x}=\gamma^{\beta}(l) w, & x=L .\end{cases}
$$

Next, to remove the advective term in the interval $(0, L)$, we multiply the differential equation by $e^{-c x}$ and note that

$$
\left(e^{-c x} w_{x}\right)_{x}=e^{-c x} w_{x x}-c e^{-c x} w_{x}=e^{-c x}\left(w_{x x}-c w_{x}\right) .
$$

Thus, we obtain the two systems

$$
\begin{cases}\left(e^{-c x} w_{x}\right)_{x}-g(x) e^{-c x} w=\lambda e^{-c x} w, & 0<x<L, \\ e^{-c x} w_{x}-\gamma_{0}^{\alpha} e^{-c x} w=0, & x=0, \\ e^{-c x} w_{x}-\gamma_{0}^{\beta} e^{-c x} w=0, & x=L,\end{cases}
$$

758 and

$$
\begin{cases}\left(e^{-c x} w_{x}\right)_{x}-g(x) e^{-c x} w=\lambda e^{-c x} w, & 0<x<L, \\ e^{-c x} w_{x}-\gamma^{\alpha}(l) e^{-c x} w=0, & x=0, \\ e^{-c x} w_{x}-\gamma^{\beta}(l) e^{-c x} w=0, & x=L .\end{cases}
$$

Since $e^{-c x}$ is strictly positive, systems (82) and (83) are governed by an elliptic, selfadjoint operator so that the theory from Case 1 can be applied. 


\section{B Derivation of the equation for $T(x)$}

We give a derivation of the equation for the mean first passage time function $T(x)$ from a random walk by adapting the approach in McKenzie et al. (2009) to the moving habitat.

The suitable habitat at time $t$ is the interval $[c t, L+c t]$. We assume hostile boundaries, i.e. individuals at the boundary leave the suitable habitat. We denote by $T(x, t)$ the average time that an individual located at position $x$ at time $t$ takes to reach the boundary and by $p$ the probability that the individual moves distance $\delta$ either left or right during a time step of length $\tau$

The master equation describes how $T$ changes from one time step to the next as

$$
T(x, t)=\tau+\frac{p}{2} T(x-\delta, t+\tau)+\frac{p}{2} T(x+\delta, t+\tau)+(1-p) T(x, t+\tau) .
$$

Since the habitat moves distance $c \tau$ in one time step, we have $T(\cdot, t+\tau)=T(\cdot-c \tau, t)$. Inserting this relation into the above equation and expanding the terms in Taylor series with respect to $x$, gives

$$
T=\tau+T+c \tau T^{\prime}+p \frac{\delta^{2}}{2} T^{\prime \prime}+O\left(\delta^{3}, \tau^{2}\right)
$$

Now we cancel $T$, divide by $\tau$ and take the parabolic limit $D=\lim _{\delta, \tau} \frac{p \delta^{2}}{2 \tau}$ to arrive at the 774 equation $D T^{\prime \prime}+c T^{\prime}=-1$ 